\title{
Organic molecules coming of age in quantum optics
}

\author{
Vahid Sandoghdar* \\ Max Planck Institute for the Science of Light, Staudtstraße 2, 91058 Erlangen, Germany
}

The interaction of light and matter at the nanometer scale lies at the heart of quantum optics because it concerns elementary processes such as absorption or emission of a photon by an atom. Over the past decade, we have shown that direct coupling of a photon to a single two-level atom should be possible via tight focusing. However, because transitions in quantum emitters are typically not closed, laboratory demonstrations of this idea fall short of the theoretical prediction. In this presentation I shall report on recent achievements, where the branching ratio of a single organic molecule is improved by a substantial Purcell effect when coupled to a microcavity. Furthermore, we will discuss coherent linear and nonlinear experiments on molecules coupled to subwavelength waveguides on a chip. Together with their ability to generate narrowband stream of single photons, these developments make organic molecules viable candidates for integration in chip-based quantum optical circuits.

\footnotetext{
*Corresponding author: vahid.sandoghdar@mpl.mpg.de
} 